\title{
Does Neoadjuvant Chemotherapy Increase the Survival in Patients with Locally Advanced Gastric Cancer Patients? - A Real-World Evidence
}

\begin{abstract}
Background: In locally advanced gastric cancer (LAGC), perioperative chemotherapy has shown to improve the survival to a larger extent compared to surgery alone. In India, the treatment followed for gastric carcinoma widely varies based on the type of health-care provider and treatment access. There is a paucity of data on the role of neoadjuvant chemotherapy on survival among LAGC patients in the Indian context. Aim: The aim of this study was to compare the disease-free survival (DFS) and overall survival (OS) between neoadjuvant and adjuvant chemotherapies among LAGC patients. Subjects and Methods: This was a retrospective cohort study involving clinical record review of LAGC patients enrolled between 2015 and 2017 from four tertiary cancer centers in South India. The date for the following events, namely diagnosis, recurrence, death, and last day of visit, was extracted in a mobile-based open-access tool. The median duration of OS and DFS between the neoadjuvant and adjuvant groups was compared using Kaplan-Meier survival curves. Results: Of the 137 patients, $70(51 \%)$ had received neoadjuvant chemotherapy followed by surgery and $67(49 \%)$ had adjuvant chemotherapy following the surgery. The mean (standard deviation) age of participants was 55.4 (11.4) years. Seventy-eight percent of the patients were diagnosed at Stage 3 or 4. Regional lymph nodes were involved in $83.9 \%$. The median duration of follow-up was 15 months. The OS in the neoadjuvant and adjuvant groups was 18.6 months and 8.3 months, respectively. Nonregional lymph node involvement and adjacent organ involvement had independently increased the risk of death. Conclusion: Among LAGC patients, the neoadjuvant chemotherapy indicated a better median and DFS compared to the adjuvant group. However, these findings were statistically not significant. The current study has contributed an important finding to the existing evidences of clinical practice in an Indian setting. Further large-scale studies are required to validate the promising trend of using neoadjuvant chemotherapy in LAGC.
\end{abstract}

Keywords: Collaborative Medical Oncology Group, D2 lymphadenectomy, gastric carcinoma, stomach neoplasm, Structured Operational Research and Training Initiative

\section{Introduction}

Globally, gastric cancer ranks the fourth most common cancer and second most common cause of cancer-related mortality. Among all cancer-related deaths, $8.2 \%$ of deaths occurred due to gastric cancer. ${ }^{[1]}$ Despite the annual $1.45 \%$ decrease in the incidence of gastric cancers, every year, an estimated one million gastric carcinomas are diagnosed worldwide ${ }^{[2]}$ and are accountable for 783,000 deaths. More than $50 \%$ of the new cases of gastric carcinoma occur in developing countries. ${ }^{[3]}$ The recent Indian Council of Medical Research (ICMR) report based on Indian cancer registry has estimated the incidence of gastric cancer to be approximately 34,000 which is predicted to become 50,000 by $2020 .^{[2]}$ Approximately

\footnotetext{
This is an open access journal, and articles are distributed under the terms of the Creative Commons Attribution-NonCommercial-ShareAlike 4.0 License, which allows others to remix, tweak, and build upon the work non-commercially, as long as appropriate credit is given and the new creations are licensed under the identical terms.
}

For reprints contact: WKHLRPMedknow_reprints@wolterskluwer.com seven out of ten cases are diagnosed at an advanced stage. ${ }^{[1]}$ The standard treatment for gastric cancer is complete curative resection of the tumor with a standardized D2 lymphadenectomy. ${ }^{[3]}$ Despite curative resection, nearly $50 \%$ of the patients recur with a median survival of 12 months. ${ }^{[4,5]}$

Chemotherapy given during the perioperative period (neoadjuvant and adjuvant chemotherapies) was found to influence the recurrence pattern and survival in locally advanced gastric cancer (LAGC) patients. ${ }^{[6,7]}$ Neoadjuvant chemotherapy may potentially downstage the tumor, treat the micrometastasis, and prevent the new onset of metastatic lesions. ${ }^{[8]}$ Evidence shows that both peri- and postoperative chemotherapies may increase the disease-free survival (DFS) and overall survival (OS) in

How to cite this article: Janarthinakani M,
Kalaiselvi S, Priyadarshini R, Arun S, Shashidhar K,
KrishnakumarR, etal. Does neoadjuvant chemotherapy
increase the survival in patients with locally advanced
gastric cancer patients? - A real-world evidence.
Indian J Med Paediatr Oncol 2020;41:832-40.

\section{Murugesan Janarthinakani ${ }^{1}$, Selvaraj Kalaiselvi², Rajamani \\ Priyadarshini ${ }^{3}$, Seshachalam Arun ${ }^{4}$, K Shashidhar ${ }^{5}$, R Krishnakumar, N Manjunath ${ }^{5}$, Sirigeri Roopa?, SG Raman ${ }^{1}$}

${ }^{1}$ Department of Medical Oncology, Madras Cancer Care Foundation, Chennai, Tamil Nadu, India, ${ }^{2}$ Department of Community Medicine, AIIMS, Nagpur, Maharashtra, India, ${ }^{3}$ Department of Research, Fenivi Research Solutions Private Limited, Chennai, Tamil Nadu, India, ${ }^{4}$ Department of Medical Oncology, GVN Cancer Institute, Trichy, Tamil Nadu, India,

${ }^{5}$ Vydehi Institute of Medical Sciences and Research Centre, Bengaluru, Karnataka, India, ${ }^{6}$ Meenakshi Mission Hospital and Research Centre, Madurai, Tamil Nadu, India, 'Department of Oncology, Columbia Asia, Bengaluru, Karnataka, India

Submitted: 24-Apr-2020 Revised: 06-Jul-2020 Accepted: 23-Oct-2020 Published: 31-Dec-2020

Address for correspondence: Dr. Murugesan Janarthinakani, Department of Medical Oncology, Madras Cancer Care Foundation, Chennai, Tamil Nadu, India.

E-mail: mjanarthinakani@ yahoo.com

Access this article online Website: www.ijmpo.org DOI: 10.4103/ijmpo.ijmpo_188_20 Quick Response Code:

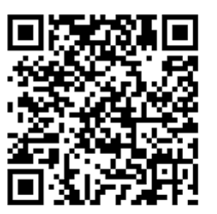


LAGC patients. ${ }^{[9,10]}$ Evidence by Cunningham et al. based on perioperative chemotherapy trial had shown the 5-year survival rate of $36 \%$ for perioperative chemotherapy arm compared to $23 \%$ survival among patients who underwent surgery alone. ${ }^{[11]}$

Depending on the extent of the disease and the patient tolerance level, perioperative chemotherapy is given alone or in combinations with radiotherapy. ${ }^{[12]}$ However, there is a regional difference in the preferred chemotherapy regimen in India due to various factors such as poor access to regional cancer centers (catering to large population), physician preference, affordability issues, and different clinical circumstances. There are several approaches being followed by health-care providers. There is a paucity of evidence in the Indian context, whether these varying treatment approaches with or without neoadjuvant chemotherapy will make a difference in disease progression and survival. ${ }^{[13]}$ The recent ICMR guidelines emphasized the lack of quality evidence on neoadjuvant regimens to guide the standard of care. ${ }^{[14]}$

Hence, the present study was conducted to compare the effectiveness of neoadjuvant chemotherapy to adjuvant chemotherapy among patients with locally advanced stomach cancer in terms of DFS and OS in selected tertiary care cancer centers in South India.

\section{Subjects and Methods}

\section{Study design}

This was a retrospective multicentric cohort study involving the review of patients' clinical records.

\section{Study setting}

This study was conducted across four centers in South India. These study sites are functioning as corporate hospitals, and the treatment-related expenditures are paid by the patient. As a part of the hospital information system, these centers maintain the patient demographic and clinical characteristics in an electronic as well as paper-based format. The patient management group involves a team of multiple specialists including a medical oncologist, radiation oncologist, surgical oncologist, and surgical gastroenterologist.

The National Comprehensive Cancer Network guidelines are widely followed with the discretion of treating physicians. The process involved in patient care management for locally advanced gastric carcinoma is depicted in Figure 1. The figure explains the chemotherapy types, adjuvant and neoadjuvant chemotherapies, and the duration. The various regimens used in the study are epirubicin + oxaliplatin + capecitabine (EOX), capecitabine + oxaliplatin, 5-fluorouracil (5-FU) + leucovorin calcium, epirubicin + Adriamycin + cisplatin $+5-\mathrm{FU}$, cisplatin +5 -FU, and 5-FU + leucovorin + oxaliplatin + docetaxel. All the node-positive patients had received radiotherapy with the discretion of a multidisciplinary tumor board.

\section{Study population}

The study population included all locally advanced gastric carcinomas (Stage 2 or more) registered for treatment from January 2015 to December 2017 and attended a minimum one follow-up visit after 3 months of treatment in the abovementioned study sites. All eligible patients were followed till February 28, 2019. Patients with metastatic stomach cancer and those who did not undergo gastrectomy or with a previous history of chemoradiotherapy were excluded from the analysis.

\section{Data collection}

From each study site, investigators extracted the data in a structured data extraction pro forma. The pro forma included patient characteristics such as age, gender, stage of disease-based computed tomography abdomen and

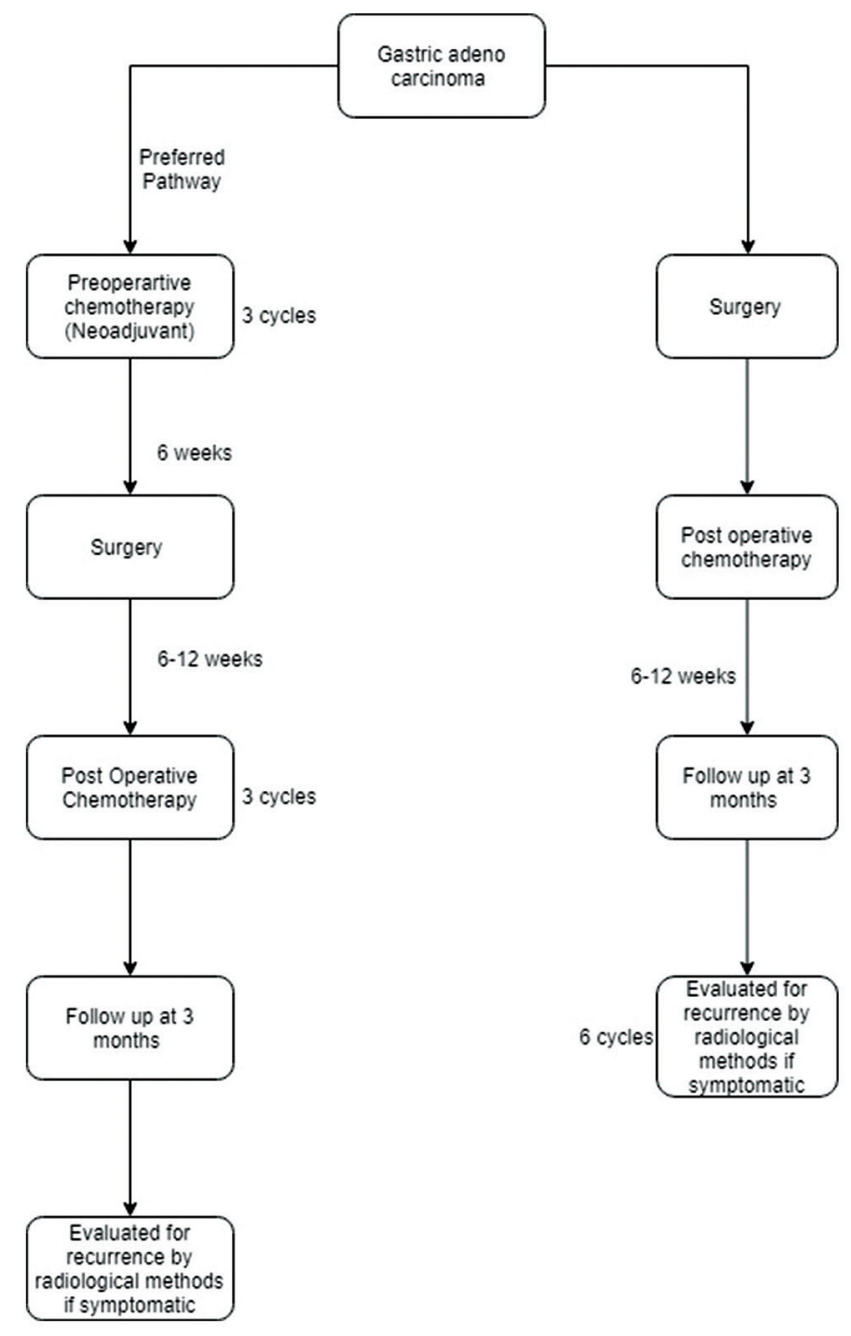

Figure 1: Process involved in patient care management for the locally advanced gastric cancer patients who underwent neoadjuvant and adjuvant chemotherapies during 2015-2017 
pelvis, and histopathological examinations. The study also included type of surgery, chemotherapy regimen used, timing of chemotherapy in relation to surgery (neoadjuvant or adjuvant) and number of cycles given, adverse events, date of each visit, and date of recurrence. The definition of the terms used in the present study such as lost to follow-up, radiological response, DFS, and OS is given in Box 1.

\section{Data analysis}

Data captured in data extraction pro forma were entered in EpiCollect5 (Imperial College, London). Data were analyzed using Stata (version 14, University of Texas, StataCorp). Continuous variables such as age and tumor size are summarized as mean (standard deviation [SD]). Clinical staging, comorbidities, grade of tumor, and chemoresponses are summarized in terms of frequencies and percentages. Outcomes considered in this study are DFS after surgery and OS after the diagnosis. The database was frozen on February 28, 2019. The duration of survival and DFS is summarized as a median. The difference in duration of DFS was initially analyzed through Kaplan-Meier survival analysis using log-rank test. The hazard ratio (HR) adjusted for background characteristics such as age group, staging, grade of tumor, and mode of treatment was estimated using the Cox proportional hazard model. Factors associated with overall and DFS are presented as adjusted HR (aHR) with a $95 \%$ confidence interval (CI).

\section{Ethics approval}

Administrative approval was obtained from all the participating institutes for accessing data. Ethics approval was obtained from the Institutional Ethics Committee (IEC) of the GVN Cancer Institute (dated July 30, 2018), Trichy, and the Ethics Advisory Group of the International Union Against Tuberculosis and Lung Disease, Paris, France (EAG number 30/18). As the study involves a review of patient records (secondary data), a waiver for informed consent was sought, and the same was approved by the IEC.

\section{Results}

\section{Patient characteristics}

A total of 137 patients with LAGC were enrolled in the study. Of the 137 patients, $94(69.3 \%)$ were men. The mean (SD) age was 55.4 (11.4) years, and one-third (35\%) were above 60 years. The body of the stomach was the most common site of cancer $(23.6 \%)$, followed by antrum and pylorus $(\sim 17 \%)$. About $20 \%$ had a tumor at the esophagogastric junction or cardia. Seventy-eight percent were diagnosed at late stage (third or fourth). Regional lymph nodes were involved in $83.9 \%$ of the patients. Except for the site of tumor and number of nodal sites involved, other patient demographic and clinical characteristics were found to be similar [Table 1]. Tumor at upper one-third of the stomach (cardia, gastroesophageal junction, and fundus) was more frequently observed in the neoadjuvant group compared to the adjuvant group (64.9\% vs. $17.9 \%, P<0.0001)$.

\section{Treatment}

Of the 137 patients, 51\% had received chemotherapy followed by surgery (neoadjuvant) and the remaining had undergone surgery directly. The common chemotherapy regimens given during the neoadjuvant phase were EOX $(n=36 ; 51.4 \%)$ and epirubicin + Adriamycin + cisplatin +5 -FU $(n=30 ; 42.9 \%)$. After surgery, $80 \%$ received chemotherapy (adjuvant), of which $25 \%$ had received oxaliplatin with capecitabine-based regimen. About 68 patients $(49.6 \%)$ had undergone partial gastrectomy, and it was more in the adjuvant group $(70 \%)$ compared to the neoadjuvant group $(30 \%)$. Overall, $89 \%$ had D2 node dissection during surgery. The mean (SD) number of chemotherapy cycles received in the neoadjuvant group was more compared to the adjuvant

\section{Box 1: Operational definition}

Locally advanced gastric carcinoma: Stage II-III as per AJCC staging manual 2017

Loss to follow-up: The time point after receiving either of the modalities is 6 months. When the study participant does not turn up for 6 months, it will be considered as loss to follow up

Radiological response

Complete response: Disappearance of all target lesions

Partial response: $>30 \%$ decrease in the sum of the longest diameters of target lesions compared with baseline

Stable disease: Neither partial response nor progressive disease

Progressive disease: $>20 \%$ increase in the sum of the longest diameter of target lesions compared with the smallest sum longest diameter recorded or appearance of one or more new lesions

Regional lymph nodes: Perigastric, paracardial, suprapyloric, infrapyloric, left gastric, celiac, common hepatic, hepatoduodenal, splenic hilar lymph nodes

Disease-free survival: Duration between the date of surgery and onset of new symptoms, clinical manifestations, radiological diagnosis, or cancer-related death

Overall survival: Duration between the date of diagnosis and day of death or last day of follow up whichever is later

AJCC - American Joint Committee on Cancer 


\begin{tabular}{|c|c|c|c|}
\hline Characteristics & Neoadjuvant therapy group, $n(\%)$ & Adjuvant therapy group, $n(\%)$ & $P$ \\
\hline Total & $70(100)$ & $67(100)$ & \\
\hline \multicolumn{4}{|l|}{ Gender } \\
\hline Male & $53(75.7)$ & $42(62.7)$ & \multirow[t]{2}{*}{0.098} \\
\hline Female & $17(24.3)$ & $25(37.3)$ & \\
\hline \multicolumn{4}{|l|}{ Comorbidities } \\
\hline Diabetes & $13(18.6)$ & $11(16.4)$ & 0.740 \\
\hline Hypertension & $13(18.6)$ & $11(16.4)$ & 0.740 \\
\hline Coronary artery disease & $2(2.9)$ & $5(7.5)$ & 0.268 \\
\hline Others & 0 & $3(4.5)$ & 0.114 \\
\hline \multicolumn{4}{|l|}{ Stage } \\
\hline Second stage & $13(18.5)$ & $17(25.4)$ & \multirow[t]{3}{*}{0.520} \\
\hline Third stage & $51(72.9)$ & $45(67.2)$ & \\
\hline Fourth stage & $6(8.6)$ & $5(7.5)$ & \\
\hline \multicolumn{4}{|l|}{ Tumor site } \\
\hline Cardia & $23(32.9)$ & $4(6.0)$ & $0.001 *$ \\
\hline Fundus & $16(22.9)$ & $5(7.5)$ & 0.012 \\
\hline Body & $25(35.7)$ & $25(37.3)$ & 0.846 \\
\hline Antrum & $20(28.6)$ & $18(26.9)$ & 0.824 \\
\hline Pylorus & $13(18.6)$ & $22(32.8)$ & $0.055^{*}$ \\
\hline Lesser curvature & $14(20.0)$ & $4(6.0)$ & $0.021 *$ \\
\hline Greater curvature & $6(8.6)$ & $2(3.0)$ & 0.275 \\
\hline Gastroesophageal junction & $11(15.7)$ & $3(4.5)$ & $0.046^{*}+x-2 x-1$ \\
\hline \multicolumn{4}{|l|}{ Number of tumor sites } \\
\hline One & $35(50.0)$ & $53(79.1)$ & \multirow[t]{4}{*}{$0.001 *$} \\
\hline Two & $17(24.3)$ & $13(19.4)$ & \\
\hline Three & $14(20.0)$ & 0 & \\
\hline More than three & $4(5.7)$ & $1(1.5)$ & \\
\hline Tumor size mean (standard deviation) & $4.88(2.17)$ & $5.89(3.62)$ & 0.070 \\
\hline \multicolumn{4}{|l|}{ Lymph node involvement } \\
\hline Nil & $5(7.2)$ & $8(12.5)$ & \multirow[t]{3}{*}{0.494} \\
\hline Regional nodes & $62(89.9)$ & $53(82.8)$ & \\
\hline Nonregional lymph nodes & $2(2.9)$ & $3(4.7)$ & \\
\hline \multicolumn{4}{|l|}{ Preoperative imaging } \\
\hline Yes & $64(97.0)$ & $52(96.3)$ & \multirow[t]{2}{*}{1.000} \\
\hline No & $2(3.0)$ & $2(3.7)$ & \\
\hline
\end{tabular}

group (5.6 [1.2] vs. 4.3 [2.4]; $P<0.001$ ). Patients who completed all six cycles of chemotherapy were more in the neoadjuvant group (80.3\%) compared to the adjuvant group (50.3). The rate of complete surgical resection (R0 resection: $97 \%$ and 91.7\%) was similar in both the neoadjuvant and adjuvant groups. Forty percent received radiotherapy after surgery, and it was comparable in both the groups $(37 \%$ vs. $41 \%)$. Patients with pN0 were significantly more in the neoadjuvant group (29\% vs. $9 \%$, $P=0.02)$ [Table 2].

\section{Outcome}

The median duration of follow-up was 15.5 months, with an interquartile range of 6.6-26.6 months. The recurrence rate was comparable in both the groups (neoadjuvant: 25.6/1000 person-years and adjuvant: 19.2/1000 person-years, $P>0.05)$. The median duration of DFS was 13.3 months in the neoadjuvant group and 10.3 months in the adjuvant group, both of which were not statistically significant [Table 3]. Similarly, the median duration of survival was more in the neoadjuvant group (18.6 months) compared to the adjuvant group (8.3 months) but without statistical significance [Figure 2]. When the DFS was compared across several characteristics, surgical staging, grade of tumor (Grade 3 [aHR: 2] and Grade 4 [aHR: 8.6]), and nonregional lymph node involvement (aHR: 26.6) were found to independently increase the risk of recurrence [Table 4]. Similarly, nonregional lymph node involvement and adjacent organ involvement had independently increased the risk of death. Patients who received at least four cycles of chemotherapy had aHR of 0.28 (95\% CI: $0.11,0.70$; $P=0.006$ ), and in those who received more than 6 cycles, it was 0.19 (95\% CI: $0.08-0.46 ; P=0.001)$ [Table 5]. 


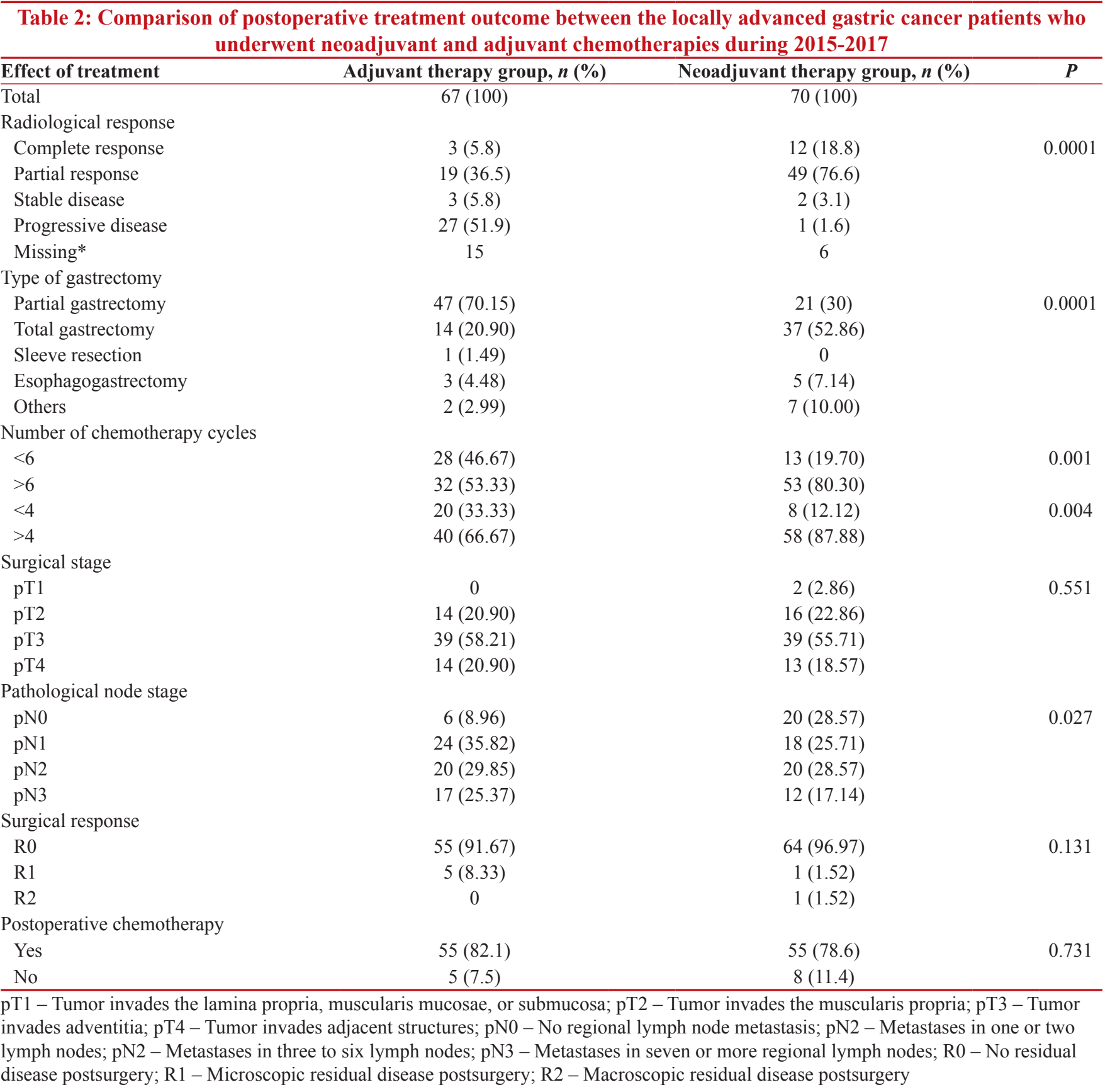

\section{Discussion}

The present study is one of the very few studies to congregate evidence and to compare the treatment outcomes of neoadjuvant and adjuvant therapies in locally advanced gastric carcinoma among the Indian population. The present study shows better OS rates and DFS rates in the neoadjuvant group as compared to the adjuvant group. The high pathological staging, tumor grade, and nonregional lymph nodal involvement had independently increased the risk of recurrence and death.

In terms of absolute number of recurrences, the neoadjuvant group had more recurrences. Though it seems to be contradictory to the expected low recurrence in the neoadjuvant group, it is possible due to the following reasons: (1) outcome in cancer survival studies includes the number of events and also the time taken to develop that event. (2) As the survival is more in the neoadjuvant group, the recurrences also logically will be more in the neoadjuvant group.

The study conducted by Cunningham et al. (MAGIC trial) ${ }^{[11]}$ has been the pioneering trial that paved the way for administering chemotherapy along with surgery in clinical practice. The 2-year survival documented in the MAGIC trial was $50 \%$ and $41 \%$ among the neoadjuvant and adjuvant groups, respectively, whereas in our study, it was $81 \%$ and $77 \%$, respectively. This could be due to 


\begin{tabular}{|c|c|c|}
\hline Estimate & Adjuvant chemotherapy $(n=67)$ & Neoadjuvant chemotherapy $(n=70)$ \\
\hline \multicolumn{3}{|l|}{ Overall survival } \\
\hline Median overall survival time (months) & 8.3 & 18.6 \\
\hline Number of deaths & 8 & 12 \\
\hline Duration followed (months) & 919.5 & 1440.7 \\
\hline Incidence rate for overall survival & $8.47 / 1000$ person-months & $8.33 / 1000$ person-months \\
\hline $\mathrm{HR}$ & Reference & 0.97 (95\% CI: $0.39-2.37)$ \\
\hline \multicolumn{3}{|l|}{ Disease-free survival } \\
\hline Median disease-free survival (months) & 10.3 & 13.3 \\
\hline Number of recurrence & 15 & 26 \\
\hline Duration followed (months) & 780.2 & 1014 \\
\hline Incidence rate for disease-free survival & $19.2 / 1000$ person-months & $25.6 / 1000$ person-months \\
\hline HR & Reference & 1.25 (95\% CI: 0.66-2.37) \\
\hline
\end{tabular}

CI - Confidence interval; HR - Hazard ratio

Table 4: Disease-free survival of the locally advanced gastric cancer patients who underwent neoadjuvant and adjuvant chemotherapies during 2015-2017

\begin{tabular}{|c|c|c|c|c|c|c|c|}
\hline Variables & Categories & $\mathbf{H R}^{\wedge}$ & 95\% CI & $P$ & $\mathbf{H R}^{\wedge \wedge}$ & $95 \%$ CI & $P$ \\
\hline \multirow[t]{2}{*}{ Group } & Adjuvant & Reference & & & Reference & & \\
\hline & Neoadjuvant & 1.25 & $0.66-2.37$ & 0.49 & 1.46 & $0.64-3.32$ & 0.372 \\
\hline \multirow[t]{2}{*}{ Sex } & Male & Reference & & & Reference & & 0.100 \\
\hline & Female & 0.58 & $0.28-1.22$ & 0.15 & 0.44 & $0.17-1.17$ & \\
\hline \multirow[t]{3}{*}{ Stage } & 2 & Reference & & & & & \\
\hline & 3 & 1.55 & $0.6-4.0$ & 0.36 & - & - & - \\
\hline & 4 & 2.73 & $0.57-10.4$ & 0.23 & - & - & - \\
\hline \multirow{3}{*}{$\begin{array}{l}\text { Lymph node } \\
\text { involvement }\end{array}$} & Nil & Reference & & & 2.30 & $0.31-17.08$ & 0.418 \\
\hline & Regional nodes & 2.04 & $0.40-8.5$ & 0.33 & 63.65 & $2.76-1465.90$ & 0.009 \\
\hline & Nonregional lymph nodes & 26.6 & $3.1-225.5$ & $0.003 *$ & Reference & & \\
\hline \multirow{2}{*}{$\begin{array}{l}\text { Adjacent organ } \\
\text { involvement }\end{array}$} & No & Reference & & & - & - & - \\
\hline & Yes & 0.92 & $1.3-6.8$ & 0.94 & - & - & - \\
\hline \multirow{4}{*}{$\begin{array}{l}\text { Radiological } \\
\text { response }\end{array}$} & Complete response & Reference & & & - & - & - \\
\hline & Partial response & 1.67 & $0.57-4.90$ & 0.35 & - & - & - \\
\hline & Progressive disease & 1.58 & $0.29-8.77$ & 0.6 & - & - & - \\
\hline & Stable disease & 1.44 & $0.45-4.62$ & 0.54 & - & - & - \\
\hline \multirow[t]{2}{*}{ Node dissection } & D1 & Reference & & & Reference & & \\
\hline & D2 & 0.14 & $0.02-1.18$ & 0.07 & 0.99 & $0.05-21.59$ & 0.998 \\
\hline Surgical stage ${ }^{\#}$ & & 1.29 & $0.79-2.12$ & 0.3 & & & \\
\hline \multirow{4}{*}{$\begin{array}{l}\text { Pathological nodal } \\
\text { stage }\end{array}$} & $\mathrm{pN} 0$ & Reference & & & Reference & & \\
\hline & $\mathrm{pN} 1$ & 0.83 & $0.31-2.25$ & 0.72 & 0.76 & $0.18-3.26$ & 0.711 \\
\hline & $\mathrm{pN} 2$ & 0.86 & $0.33-2.25$ & 0.77 & 0.76 & $0.17-3.41$ & 0.722 \\
\hline & $\mathrm{pN} 3$ & 1.33 & $0.53-3.30$ & 0.54 & 1.29 & $0.29-5.67$ & 0.735 \\
\hline \multirow[t]{3}{*}{ Surgical response } & R0 & Reference & & & Reference & & \\
\hline & R1 & 1.34 & $0.48-3.83$ & 0.56 & 3.66 & $0.95-14.12$ & 0.059 \\
\hline & $\mathrm{R} 2$ & 8.66 & $1.1-6.70$ & $0.04 *$ & - & - & - \\
\hline \multirow{5}{*}{$\begin{array}{l}\text { Grade of tumor }{ }^{\#} \\
\text { Number of } \\
\text { chemotherapy cycles }\end{array}$} & & 1.67 & $0.98-2.85$ & 0.06 & - & - & - \\
\hline & $<6$ & Reference & & & & & \\
\hline & $>6$ & 0.69 & $0.32-1.51$ & 0.35 & - & - & - \\
\hline & $<4$ & Reference & & & - & - & - \\
\hline & $>4$ & 0.87 & $0.34-2.24$ & 0.78 & - & - & - \\
\hline
\end{tabular}

${ }^{\wedge}$ Unadjusted risk ratio; ${ }^{*} P<0.05 ;{ }^{\wedge}$ Adjusted HR; ${ }^{*}$ Risk progression from Stage $1 /$ Grade 1 to one unit increase in subsequent categories. $\mathrm{CI}$ - Confidence interval; HR - Hazard ratio; pT1 - Tumor invades the lamina propria, muscularis mucosae or submucosa; pT2 - Tumor invades the muscularis propria; pT3 - Tumor invades adventitia; pT4 - Tumor invades adjacent structures; pN0 - No regional lymph node metastasis; pN2 - Metastases in one or two lymph nodes; pN2 - Metastases in three to six lymph nodes; pN3 - Metastases in seven or more regional lymph nodes; R0 - No residual disease postsurgery; R1 - Microscopic residual disease postsurgery; R2 - Macroscopic residual disease postsurgery 


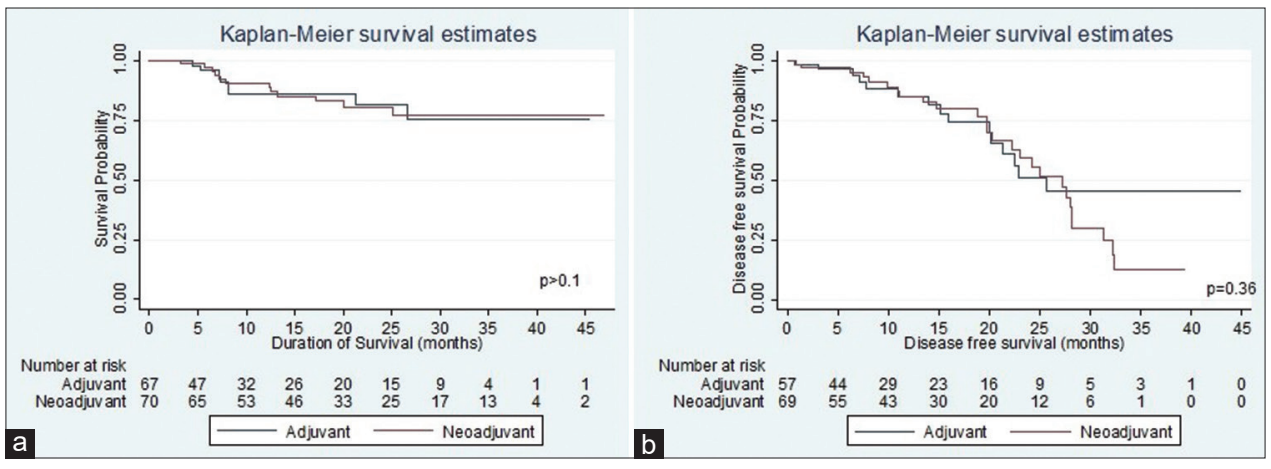

Figure 2: (a) Comparison of overall survival between neoadjuvant and adjuvant chemotherapies in locally advanced gastric carcinoma, South India 2015-2017. (b) Comparison of disease-free survival between neoadjuvant and adjuvant chemotherapies in locally advanced gastric carcinoma, South India 2015-2017

Table 5: Overall survival of the locally advanced gastric cancer patients who underwent neoadjuvant and adjuvant chemotherapies during 2015-2017

\begin{tabular}{|c|c|c|c|c|c|c|c|}
\hline Variables & Categories & $\overline{\mathbf{H R}^{\wedge}}$ & $95 \% \mathrm{CI}$ & $P$ & $\mathbf{H R}^{\wedge \wedge}$ & $95 \% \mathrm{CI}$ & $P$ \\
\hline \multirow[t]{2}{*}{ Group } & Adjuvant & Reference & & & Reference & & \\
\hline & Neoadjuvant & 0.97 & $0.39-2.372$ & 0.94 & 1.05 & $0.36-3.00$ & 0.933 \\
\hline \multirow[t]{2}{*}{ Sex } & Male & Reference & & & Reference & & \\
\hline & Female & 0.25 & $0.06-1.07$ & 0.06 & 0.12 & $0.01-1.06$ & 0.056 \\
\hline \multirow[t]{3}{*}{ Stage } & 2 & Reference & & & & & \\
\hline & 3 & 0.73 & $0.24-2.23$ & 0.59 & - & - & - \\
\hline & 4 & 1.07 & $0.19-5.83$ & 0.94 & - & - & - \\
\hline \multirow{3}{*}{$\begin{array}{l}\text { Lymph node } \\
\text { involvement }\end{array}$} & Nil & Reference & & & Reference & & \\
\hline & Regional nodes & 1.12 & $0.15-8.5$ & 0.9 & 0.72 & $0.09-5.87$ & 0.757 \\
\hline & Nonregional lymph nodes & 20.8 & $2.18-197.9$ & $0.008^{*}$ & 9.14 & $0.71-117.4$ & 0.089 \\
\hline \multirow{2}{*}{$\begin{array}{l}\text { Adjacent organ } \\
\text { involvement }\end{array}$} & No & Reference & & & Reference & & \\
\hline & Yes & 5.75 & $1.67-19.8$ & $0.006^{*}$ & 0.19 & $0.05-0.80$ & 0.023 \\
\hline \multirow{4}{*}{$\begin{array}{l}\text { Radiological } \\
\text { response }\end{array}$} & Complete response & Reference & & & - & - & - \\
\hline & Partial response & 1.54 & $0.34-7.04$ & 0.58 & & & \\
\hline & Progressive disease & 4.1 & $0.57-29.4$ & 0.16 & & & \\
\hline & Stable disease & 0.64 & $0.09-4.53$ & 0.653 & & & \\
\hline \multirow[t]{2}{*}{ Node dissection } & D1 & Reference & & & - & - & - \\
\hline & D2 & 0.36 & $0.05-2.78$ & 0.3 & & & \\
\hline \multirow{4}{*}{ Surgical stage } & $\mathrm{pT} 1$ & Reference & & & Reference & & \\
\hline & pT2 & 0.19 & $0.03-1.15$ & 0.07 & 0.44 & $0.05-3.58$ & 0.441 \\
\hline & pT3 & 0.15 & $0.03-0.70$ & $0.02 *$ & 0.32 & $0.04-2.79$ & 0.302 \\
\hline & $\mathrm{pT} 4$ & 0.23 & $0.04-1.18$ & 0.08 & 0.24 & $0.03-1.98$ & 0.184 \\
\hline \multirow{4}{*}{$\begin{array}{l}\text { Pathological } \\
\text { nodal stage }\end{array}$} & $\mathrm{pN} 0$ & Reference & & & - & - & - \\
\hline & pN1 & 0.14 & $0.03-0.66$ & $0.01 *$ & & & \\
\hline & $\mathrm{pN} 2$ & 0.48 & $0.18-1.34$ & 0.16 & & & \\
\hline & $\mathrm{pN} 3$ & 0.23 & $0.06-0.87$ & $0.03 *$ & & & \\
\hline \multirow{5}{*}{$\begin{array}{l}\text { Grade of tumor } \\
\text { Number of } \\
\text { chemotherapy } \\
\text { cycles }\end{array}$} & & 0.61 & $0.29-1.30$ & 0.2 & & & \\
\hline & $<6$ & Reference & - & & - & - & - \\
\hline & $>6$ & 0.19 & $0.08-0.46$ & $0.001^{*}$ & - & - & - \\
\hline & $<4$ & Reference & & & Reference & & \\
\hline & $>4$ & 0.28 & $0.11-0.70$ & $0.006^{*}$ & 0.36 & $0.10-1.35$ & 0.130 \\
\hline
\end{tabular}

${ }^{\wedge}$ Unadjusted risk ratio; ${ }^{*} P<0.05 ;{ }^{\wedge}$ Adjusted HR; ${ }^{*}$ Risk progression from Grade 1 to one unit increase in subsequent categories. CI $-\mathrm{Confidence}$ interval; HR -Hazard ratio; pT1 - Tumor invades the lamina propria, muscularis mucosae or submucosa; pT2 - Tumor invades the muscularis propria; pT3 - Tumor invades adventitia; pT4 - Tumor invades adjacent structures; pN0 - No regional lymph node metastasis; pN2 - Metastases in one or two lymph nodes; pN2 - Metastases in three to six lymph nodes; pN3 - Metastases in seven or more regional lymph nodes; R0 - No residual disease postsurgery; R1 - Microscopic residual disease postsurgery; R2 - Macroscopic residual disease postsurgery

better compliance with chemotherapy in our study. Further, in their study, the proportion of patients completing six cycles was $48 \%$, whereas in the present study, $67 \%$ had completed all six cycles. From the Indian context, Ostwal et al. and Chawla et al. had reported the tolerability of newer neoadjuvant chemotherapy regimen (EOX/ECF) 
in gastric cancers. The study report by Ostwal et al. had reported the median DFS and overall survival to be 31 and 37 months, respectively, in the neoadjuvant group. The survival reported in the current study is less compared to other studies reported from India. This could be due to difference in the distribution of tumor sites and attrition rate.

Our study results are in congruence with other studies like FNCLCC ${ }^{[15-17]}$ which followed the path of the MAGIC trial, testifying favorable survival outcomes for adjuvant and neoadjuvant therapies in gastric cancer. ${ }^{[18]}$ Although the FAMTX trial[19] could not demonstrate better outcomes for neoadjuvant chemotherapy, a meta-analysis on chemotherapy concluded that chemotherapy was advantageous in advanced gastric cancer. ${ }^{[20]}$

In India, there has been a long-existing disparity in gastric cancer research in general and specifically in the context of therapeutics compared to other Western and Asian countries, as reported by review articles. ${ }^{[18,21]}$ Studies have reported neoadjuvant therapy to be reliable in LAGC and improving outcomes such as resectability rate and survival while reducing recurrence. ${ }^{[22-25]}$ In the recent randomized controlled trial comparing two chemotherapy regimens in the neoadjuvant setting, the median OS at 2 years was $52 \%$ and $44 \%$ for both the groups. ${ }^{[22]}$ Kushwaha and Vidyarthi reported an improvement in resectability rate following neoadjuvant chemotherapy after 4-6 cycles. ${ }^{[26]}$ In our study, patients receiving at least four cycles of chemotherapy had a reduction in the mortality rate. Our study also found that patients receiving neoadjuvant chemotherapy had statistically insignificant longer median DFS (18.6 vs. 8.3 months) than those receiving adjuvant chemotherapy.

The treatment of LAGC is not uniform in India and varies across centers due to several patient- and health-care provider-related factors. D2 dissection is the standard surgery in gastric carcinoma among the Indian population, and in our study population, more than $80 \%$ underwent D2 dissection. ${ }^{[27]}$ In our study, patients with tumors in the cardia received neoadjuvant therapy more often than patients with pylorus tumors. As the prognosis and survival from the proximal site of gastric cancers are inferior, more representation of patients with the cardia site of gastric cancers in the neoadjuvant chemotherapy group could have precluded the statistical significance. This can be explained by the fact that most pyloric tumors present with obstructive features necessitating upfront surgery instead of neoadjuvant chemotherapy and vice versa for the patients with tumor in the cardia. ${ }^{[28,29]}$ Hence, the future randomized control trial has to plan the enrollment with adequate representation from various sites of gastric cancers such as stratified randomization techniques. Due to short follow-up and smaller sample size, the number of adverse events observed was smaller, and there was a nonuniform representation of patients in the type of nodal dissection.
Furthermore, as this was a retrospective study, missing data were to the extent of $8 \%-10 \%$.

\section{Conclusion}

In locally advanced gastric carcinoma, perioperative chemotherapy has shown to improve the survival in comparison with surgery alone. The neoadjuvant chemotherapy showed a better median overall and disease-free survival compared to the adjuvant group. Nonregional lymph node involvement and adjacent organ involvement had independently increased the risk of death.

The major limitation of the study was the small sample size which could have contributed to the statistical insignificance. However, the study has contributed important findings to the already existing global and regional evidence. In this study, we have excluded the patients who received few cycles of neoadjuvant chemotherapy but did not undergo surgery due to various reasons including loss to follow-up, disease progression, and worsening performance status. This may give rise to selection bias. However, the number excluded was minimal. Hence, this limitation is unlikely to change the survival estimates. Furthermore, in this study, a higher proportion of patients from neoadjuvant chemotherapy had a tumor at the cardiac site compared to adjuvant chemotherapy. As the prognosis of proximal site gastric cancers is expected to result in a poor survival, this could have precluded the statistical significance. Adverse events due to neoadjuvant chemotherapy can influence the survival pattern. However, this study did not account for any adverse events that occurred during the course of treatment. Despite the limitations, the current retrospective study shows a promising trend toward using neoadjuvant chemotherapy in LAGC patients. The researchers recommend similar studies in larger settings with robust randomization based on the influencing factors. High-quality evidence is the need of the hour to bring about changes in the current guidelines of gastric cancer management.

\section{Acknowledgments}

This research was conducted through the Structured Operational Research and Training Initiative (SORT IT), a global partnership led by the Special Program for Research and Training in Tropical Diseases at the World Health Organization. The training model is based on a course developed jointly by the International Union Against Tuberculosis and Lung Disease (The Union) and Medécins sans Frontières (Doctors Without Borders). The specific SORT IT program which resulted in this publication was jointly developed and implemented by Fenivi Research Solutions Private Limited, Chennai, India; The Union South-East Asia Office, New Delhi, India; and the Center for Operational Research, The Union, Paris, France. Mentorship and the coordination/facilitation of this particular SORT IT program were provided through 
Fenivi Research Solutions Private Limited, Chennai, India; The Union South-East Asia Office, New Delhi, India; the Center for Operational Research, The Union, Paris, France; Jawaharlal Institute of Postgraduate Medical Education and Research, Puducherry, India; All India Institute of Medical Sciences, Nagpur, India; and Velammal Medical College Hospital and Research Institute, Madurai, India. The specific SORT IT program which resulted in this publication was based on the data shared by the members of Collaborative Medical Oncology Group, India. Dr. Jegan Niwas Kannan and S. Mahalakshmi provided support in data extraction/analysis.

\section{Financial support and sponsorship}

Nil.

\section{Conflicts of interest}

There are no conflicts of interest.

\section{References}

1. International Agency for Research on Cancer. GLOBOCAN Cancer Fact Sheets: Stomach Cancers. International Agency for Research on Cancer; 2008.

2. Indian Council of Medical Research. Consensus Document for Management of Gastric Cancer. New Delhi: Indian Council of Medical Research; 2014.

3. Global Burden of Disease. GBD Compare IHME Viz Hub. Global Burden of Disease; 2013.

4. Sitarz R, Skierucha M, Mielko J, Offerhaus GJ, Maciejewski R, Polkowski WP. Gastric cancer: Epidemiology, prevention, classification, and treatment. Cancer Manag Res 2018;10:239-48.

5. Dikken JL, van de Velde CJ, Coit DG, Shah MA, Verheij M, Cats A. Treatment of resectable gastric cancer. Therap Adv Gastroenterol 2012;5:49-69.

6. Park SC, Chun HJ. Chemotherapy for advanced gastric cancer: Review and update of current practices. Gut Liver 2013;7:385-93.

7. Mirza A, Pritchard S, Welch I. The postoperative component of MAGIC chemotherapy is associated with improved prognosis following surgical resection in gastric and gastrooesophageal junction adenocarcinomas. Int J Surg Oncol 2013;2013:781742.

8. Glynne-Jones R, Grainger J, Harrison M, Ostler P, Makris A. Neoadjuvant chemotherapy prior to preoperative chemoradiation or radiation in rectal cancer: Should we be more cautious? Br J Cancer 2006;94:363-71.

9. Eom BW, Kim S, Kim JY, Yoon HM, Kim MJ, Nam BH, et al. Survival benefit of perioperative chemotherapy in patients with locally advanced gastric cancer: A propensity score matched analysis. J Gastric Cancer 2018;18:69-81.

10. Glatz T, Bronsert P, Schäfer M, Kulemann B, Marjanovic G, Sick O, et al. Perioperative platin-based chemotherapy for locally advanced esophagogastric adenocarcinoma: Postoperative chemotherapy has a substantial impact on outcome. Eur J Surg Oncol 2015;41:1300-7.

11. Cunningham D, Allum WH, Stenning SP, Thompson JN, Van de Velde CJ, Nicolson M, et al. Perioperative chemotherapy versus surgery alone for resectable gastroesophageal cancer. N Engl J Med 2006;355:11-20.

12. Earle CC, Maroun J, Zuraw L, Cancer Care Ontario Practice Guidelines Initiative Gastrointestinal Cancer Disease Site Group. Neoadjuvant or adjuvant therapy for resectable gastric cancer? A practice guideline. Can J Surg 2002;45:438-46.

13. Ministry of Health and Family Welfare Government of India. Achievements Under National Cancer Control Programme. Ministry of Health and Family Welfare Government of India.

14. Shrikhande SV, Sirohi B, Barreto SG, Chacko RT, Parikh PM, Pautu J, et al. Indian Council of Medical Research consensus document for the management of gastric cancer. Indian $\mathrm{J}$ Med Paediatr Oncol 2014;35:239-43.

15. Sasako M, Sakuramoto S, Katai H, Kinoshita T, Furukawa H, Yamaguchi $\mathrm{T}$, et al. Five-year outcomes of a randomized phase III trial comparing adjuvant chemotherapy with S-1 versus surgery alone in stage II or III gastric cancer. J Clin Oncol 2011;29:4387-93.

16. Bang YJ, Kim YW, Yang HK, Chung HC, Park YK, Lee KH, et al. Adjuvant capecitabine and oxaliplatin for gastric cancer after D2 gastrectomy (CLASSIC): A phase 3 open-label, randomised controlled trial. Lancet 2012;379:315-21.

17. Ychou M, Boige V, Pignon JP, Conroy T, Bouché O, Lebreton $\mathrm{G}$, et al. Perioperative chemotherapy compared with surgery alone for resectable gastroesophageal adenocarcinoma: An FNCLCC and FFCD multicenter phase III trial. J Clin Oncol 2011;29:1715-21.

18. Chelakkot PG, Ravind R, Sruthi K, Menon D. Treatment in resectable non-metastatic adenocarcinoma of stomach: Changing paradigms. Indian J Cancer 2019;56:74-80.

19. Hartgrink HH, van de Velde CJ, Putter H, Songun I, Tesselaar ME, Kranenbarg EK, et al. Neo-adjuvant chemotherapy for operable gastric cancer: Long term results of the Dutch randomised FAMTX trial. Eur J Surg Oncol 2004;30:643-9.

20. Wagner AD, Grothe W, Haerting J, Kleber G, Grothey A, Fleig WE. Chemotherapy in advanced gastric cancer: A systematic review and meta-analysis based on aggregate data. J Clin Oncol 2006;24:2903-9.

21. Sharma A, Radhakrishnan V. Gastric cancer in India. Indian J Med Paediatr Oncol 2011;32:12-6.

22. Ahmed BM, Wani KA, Wani M. OP0003 Feasibility and efficacy of neoadjuvant chemotherapy in locally advanced gastric cancer: A randomised trial. Eur J Cancer 2015;51:e1-2.

23. Chalissery JR, Jose TA, Pillai S, Unni H, Varghese KM, Gopu GP, et al. Clinical impact of adjuvant chemotherapy and radiation for carcinoma stomach: Experience from a tertiary care center. J Can Res Ther 2020.

24. Sharma A, Raina V, Lokeshwar N, Deo SV, Shukla NK, Mohanti BK. Phase II study of cisplatin, etoposide and paclitaxel in locally advanced or metastatic adenocarcinoma of gastric/ gastroesophageal junction. Indian J Cancer 2006;43:16-9.

25. Shukla NK, Deo SV, Asthana S, Raina V, Dronamaraju SS. Neoadjuvant chemotherapy in advanced gastric cancer--results of a pilot study. Trop Gastroenterol 2002;23:94-6.

26. Kushwaha AK, Vidyarthi SK. Neoadjuvant chemotherapy in locally advanced stomach cancer: Our experience. J Carcinog Mutagen 2019;10:1-2.

27. Ibrahim M, Gilbert K. Management of gastric cancer in Indian population. Transl Gastroenterol Hepatol 2017;2:64.

28. Ostwal V, Sahu A, Ramaswamy A, Sirohi B, Bose S, Talreja V, et al. Perioperative epirubicin, oxaliplatin, and capecitabine chemotherapy in locally advanced gastric cancer: Safety and feasibility in an interim survival analysis. J Gastric Cancer 2017;17:21-32.

29. Chawla T, Thambudorai R, Ashok A, Roy B, Ghosh J, Ganguly S, et al. Perioperative chemotherapy with docetaxel, oxaliplatin, fluorouracil and leucovorin (FLOT) versus epirubicin, platinum and capecitabine or flourouracil $(\mathrm{EOX} / \mathrm{ECF})$ in resectable gastric or gastroesophageal junction adenocarcinoma: Safety and response data from India. Ann Oncol 2019;30:252-324. 